\title{
SHANGHAI FEVER: A NEW PRESENTATION OF PSEUDOMONAS DISEASE IN BRAZIL
}

F. Domingues Penteado ${ }^{1}$, V. Bain ${ }^{1}$, M. Kleiman Froiman ${ }^{1}$, C.A. Paz Roman ${ }^{1}$, G. Stravinskas Durigon ${ }^{1}$, M.F. Pereira Badue ${ }^{1}$, N. Litvinov ${ }^{1}$, C. Sanson Yoshino ${ }^{1}$, H.H.

${ }^{1}$ Instituto da Criança do Hospital das Clínicas da Faculdade de Medicina da Universidade de São Paulo, Department of Pediatric Infectious Diseases, São Paulo, Brazil

\section{BACKGROUND}

Pseudomonas aeruginosa is an important agent of healthcare associated infections, especially in immunocompromised hosts.

Shanghai fever is a communityacquired enteritis associated with sepsis and isolation of Pseudomonas spp. It is a rare severe condition that affects previously healthy infants.

Most case reports are from East Asian countries. To our knowledge, we describe the first case of Shanghai Fever in Brazil.

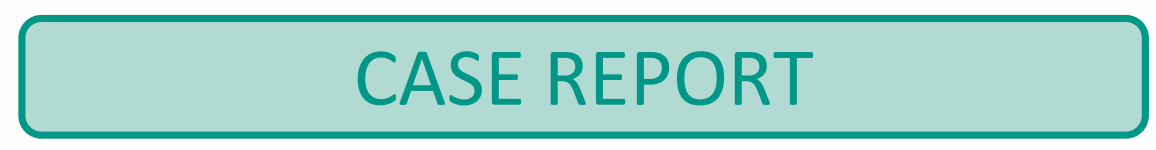

A previously healthy seven monthold boy was admitted to the intensive care unit (ICU) due to septic shock with disseminated intravascular coagulation. He had three days of fever and bloody stools. Ceftriaxone was prescribed because of suspected Salmonella enteritis.

After three days, two necrotic skin lesions suggestive of ecthyma gangrenosum were observed on his right leg and perineum. Ceftriaxone was changed to piperacilin- tazobactam for empirical Pseudomonas coverage.

On the eleventh day he presented peritonism and abdominal distension. Laparotomy revealed three bowel perforations (sigmoid colon and rectum). Colostomy was performed. Antibiotics were changed empirically to vancomycin and meropenem. He was discharged after 28 days.

Cultures from skin biopsy and peritoneal fluid yield $P$. aeruginosa. Blood cultures were negative. Primary and acquired immunodeficiencies were ruled out.

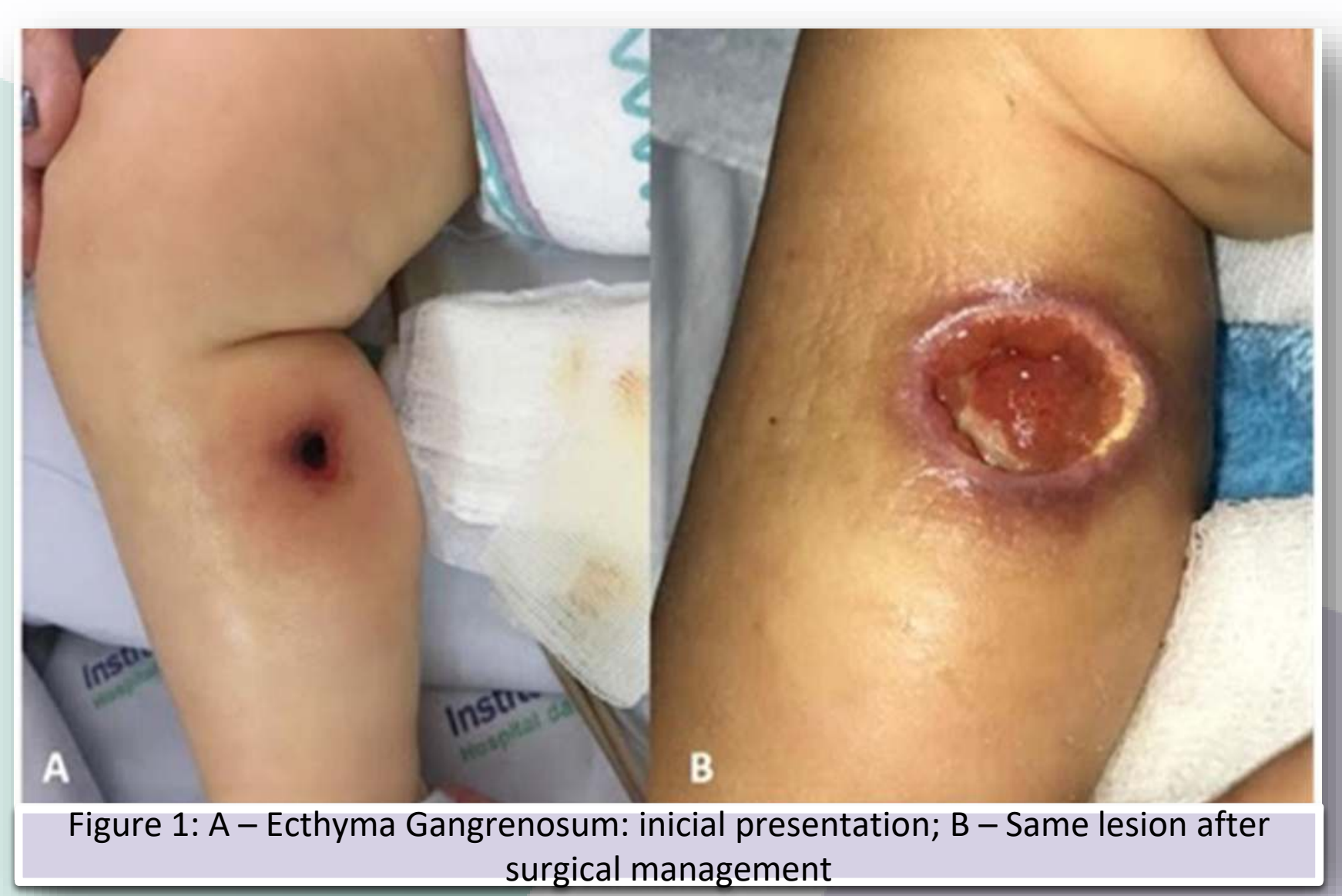

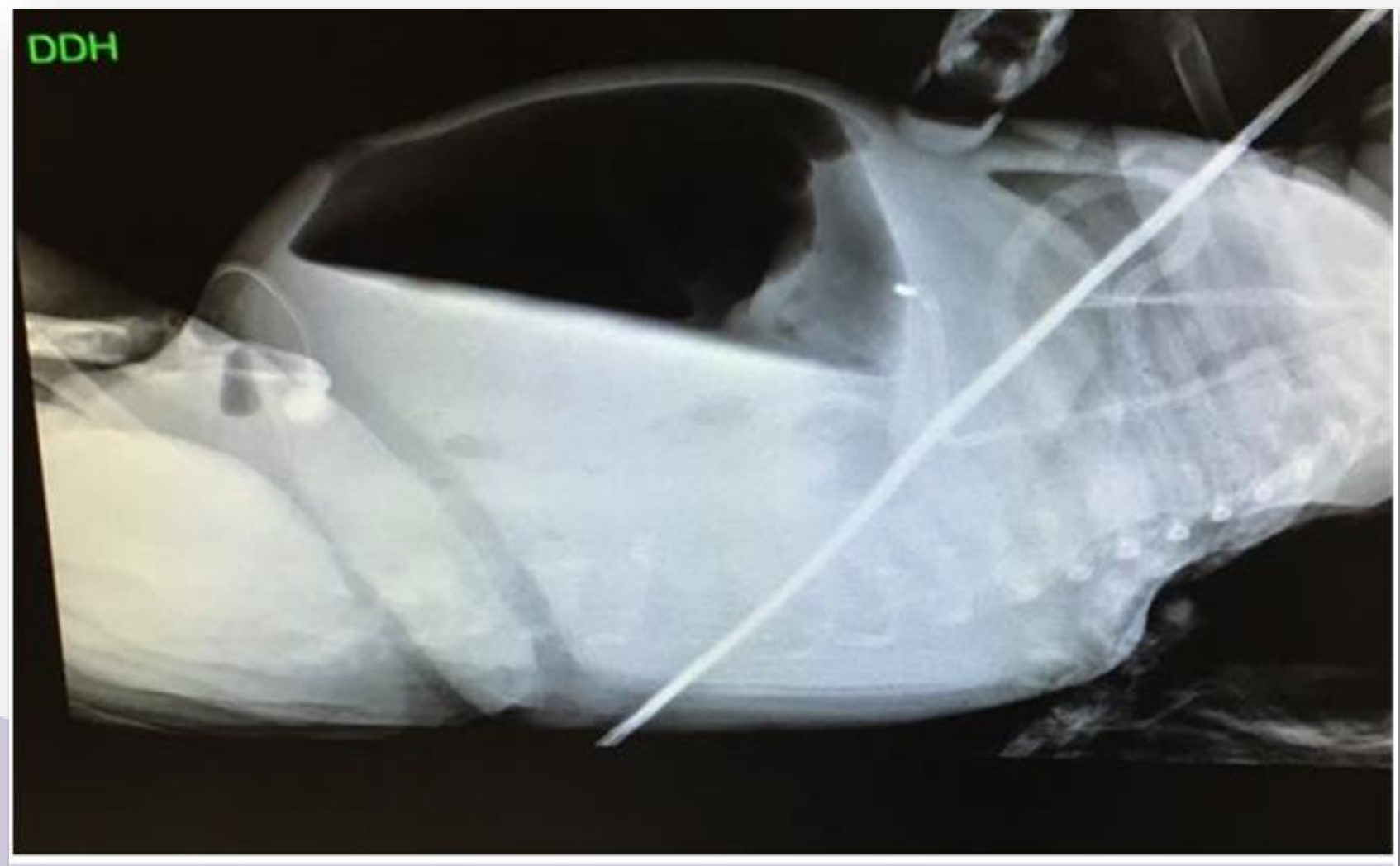

Figure 2: Pneumoperitoneum shown in abdominal radiograph (dorsal decubitus view)
Instituto da Crianca Hospital das Clinicas - FMUSP

LEARNING POINTS

The presence of Pseudomonas in stool cultures is rare and with controversial significance. In ICU setting, colonization is a late event and can be related to diarrhea without systemic symptoms. Treatment does not change outcome.

Shanghai Fever is not associated to hospital-acquired colonization, but to more virulent community strains of Pseudomonas, demonstrating higher cytotoxic and invasive profiles.

It is important to have a high level of suspicion of Pseudomonas as an agent of sepsis related to diarrhea or ecthyma gangrenosum in healthy infants not responding to standard empirical treatment.

References:

Associang CH, Janapatla RP, Wang YH, et al. Pseudomonas aeruginosa36(12): 1119-1123.

2. Chuang C, Wang Y, Chang $\mathrm{H}$, et al. Shanghai fever: a distinct Pseudomonas aeruginosa enteric disease. Gut. 2014; 63:736-743. 3. Cheng $\mathrm{Y}$, Lee $\mathrm{H}$, Yeung $\mathrm{C}$, Chan W. Clinical Significance in Previously Healthy Children of Pseudomonas aeruginosa in the Stool. Pediatr Neonatol. 2009; 50(1):13-17.

L Nosocomial Diarrhea in the 2006; 10(6): 384-389. 